\title{
Influence of Different Lateral Bending Angles on the Flow Pattern of Pumping Station Lateral Inflow
}

\author{
Jiren Zhou, ${ }^{1}$ Miaomiao Zhao, ${ }^{1}$ Chuan Wang ${ }^{(D)},{ }^{1,2}$ and Zhenjun Gao ${ }^{3}$ \\ ${ }^{1}$ College of Hydraulic Science and Engineering, Yangzhou University, Yangzhou 225009, China \\ ${ }^{2}$ Research Center of Fluid Machinery Engineering and Technology, Jiangsu University, Zhenjiang 212013, China \\ ${ }^{3}$ College of Mechanical and Power Engineering, China Three Gorges University, Yichang 443002, China
}

Correspondence should be addressed to Chuan Wang; wangchuan198710@126.com

Received 16 December 2020; Revised 5 February 2021; Accepted 2 April 2021; Published 19 April 2021

Academic Editor: Yong Zhu

Copyright ( $\odot 2021$ Jiren Zhou et al. This is an open access article distributed under the Creative Commons Attribution License, which permits unrestricted use, distribution, and reproduction in any medium, provided the original work is properly cited.

\begin{abstract}
A model of the pumping station lateral inflow forebay was established to explore the influence of different lateral bending angles of the pumping station lateral inflow. The lateral bending angles were set at $45^{\circ}$ and $60^{\circ}$, and the two schemes were calculated separately. Analyzing the results of the numerical simulation showed that the flow patterns of the diversion passages of different schemes were good, but the advancing mainstream of the l\# inlet passage near the sidewall was seriously deviated after entering the forebay. Most of the water can flow smoothly into the inlet passage, while a small part of the water flowed into the sidewall and formed a backflow, resulting in a large-scale backflow zone near the left sidewall of the forebay. Moreover, the flow in the backflow zone was turbulent, which affected the water inlet conditions of the $1 \#$ water flow passage. Comparing the water inlet conditions of the water passage with the numerical simulation results of $45^{\circ}$ and $60^{\circ}$ bending angles showed that the larger the lateral bending angle of the forebay was, the worse the flow pattern of the water flow, and the more unfavorable the pump operation.
\end{abstract}

\section{Introduction}

Irrigation and drainage pumping stations have played a vital role in fighting drought and waterlogging, ensuring increased agricultural production and income and developing the rural economy. The arrangement of several pumping stations is often restricted by objective factors such as water flow conditions and actual topography, such that the arrangement of lateral water intake has to be adopted. When water enters laterally, a certain angle is observed between the water flow direction and the forebay water flow direction, which may cause serious cavitation and vibration and will affect the normal operation of the pump. In rivers with much sediment, it will cause sedimentation and erosion of the bottom of the front pond. Therefore, taking effective rectification measures to improve the inlet flow pattern of the pumping station [1-4] is of great importance to the design and transformation of the pumping station as well as the economy and safety of the project operation.

For a long time, experts and scholars have carried out a large amount of rectification optimization research on the intake buildings of the pumping station and achieved fruitful results. Arboleda et al. [5] used the overall model test method, and the main rectification method was "block," which adjusted the flow velocity distribution of the section and weakened the lateral flow velocity in the incoming flow, thus obtaining a better rectification effect. Rajendran et al. [6] used Particle Image Velocimetry (PIV) flow field display technology to capture information such as the position of the vortex near the pump inlet and, for the first time, intuitively and quantitatively revealed the vortex's motion characteristics. Hou [7] used Computational Fluid Dynamic (CFD) methods to study the flow pattern of the pressure forebay of a pumping station, and the good agreement between the calculation results and the empirical formula showed the accuracy and reliability of the CFD calculation method. The numerical simulation results provided a basis for the hydraulic optimization design of the pressure forebay. Liu et al. [8] used gates to control the water levels of the fore and intake sump, numerically simulated the flow patterns of the fore and intake sump before and after the rectification, and concluded that a combination of several engineering 
measures such as a discontinuous sill and a pressure plate was adopted. These methods can make the flow velocity distribution of the forebay uniform and effectively improve the efficiency of the pumping station. Ansar et al. [9] took the pumping station inlet tank as the research object, compared and analyzed the numerical simulation results and the test results, and verified that the results obtained by the two methods were the same regardless of whether the pumping station enters the water directly or sideways. Moreover, the numerical calculation results had a high degree of credibility and can satisfy practical applications. Cheng et al. [10] analyzed the turbulence of the forebay caused by the movement characteristics of the curved water flow in the lateral inlet pumping station, which affected the pump inlet conditions, and calculated the 3D flow field of the rectification measures with the combined bottom sill. This approach effectively improved the water inlet conditions of the forebay. Liu et al. [11] proposed adding multiple sets of bottom sills in the forebay of a multiunit pumping station, using 3D turbulence numerical simulation calculations, and verifying them through hydraulic model tests, which largely eliminated the backflow and the vortex and substantially improved the flow pattern. The current research on hydraulic characteristics of pumping stations mainly includes theoretical analysis, numerical simulation, and model tests [12-17], each with its own advantages and disadvantages. With the rapid development of computer technology, numerical simulation has become more important in various research fields [18-24], and its research results have important reference value for practical engineering.

\section{Numerical Calculation Model and Calculation Method}

2.1. Calculation Model. The pumping station studied in this article is a pumping station hub with a lateral water intake. Three pump units are installed in the pump room. The design flow rate of each pump is $4.0 \mathrm{~m}^{3} / \mathrm{s}$. The inlet size of the flow passage is $2.36 \mathrm{~m}$ (width) $\times 1.8 \mathrm{~m}$ (length). The intake level of the pumping station is $1.9 \mathrm{~m}$, the elevation of the bottom of the drainage canal is $0.0 \mathrm{~m}$, and the elevation of the forebay bottom is $-1.0 \mathrm{~m}$. The main functions of the project are flood prevention and drainage. The left side of the pumping station is a self-draining culvert. When the pumping station is operating, the self-draining culvert control gate is closed. The total width of the outlet of the forebay is $B$, and the length from the center of the inlet of the forebay to the center of the outlet is $L$. Figure 1 shows that the plan layout of the intake building of the pumping station is mainly based on the $3 \mathrm{D}$ modeling and numerical simulation of the original design with lateral bending angles of $45^{\circ}$ and $60^{\circ}$.

\subsection{Calculation Method and Meshing. ANSYS CFX software} is used for numerical simulation, the standard $k-\varepsilon$ turbulence model is used for calculation, and the MESH software is used for unstructured meshing of the entire computational domain. The size of the grid is controlled. The selected grid shape is a tetrahedron to meet the calculation requirements. The $3 \mathrm{D}$ model grid diagram of the pumping station intake building when the lateral angle of $45^{\circ}$ is obtained is shown in Figure 2.

Taking the model with $45^{\circ}$ lateral bending angle and the hydraulic loss from the inlet of the approach passage to the outlet of the inlet passage as the characteristic parameter to select the appropriate grid number, the calculation formula is as follows:

$$
h_{f}=\frac{\left(P_{\text {in }}-P_{\text {out }}\right)}{\rho g},
$$

where $h_{f}$ is the hydraulic loss, $P_{\text {in }}$ is the total pressure at the inlet, $P_{\text {out }}$ is the total pressure at the outlet, $\rho$ is the density of water at $4^{\circ} \mathrm{C}$, and $g$ is the acceleration of gravity.

The calculation results under different grid numbers are shown in Table 1 and Figure 3. The analysis of grid independence reveals that the final number of grids is 3.58 million, which has a minimal effect.

2.3. Boundary Condition Setting. The inlet of the entire calculation domain is taken from the prototype of the diversion river to the upstream diversion passage at a $10 \mathrm{~m}$ section, which is set as the boundary condition of the mass flow, the inlet flow rate is $12 \mathrm{~m}^{3} / \mathrm{s}$, and the medium turbulence intensity $\mathrm{Tu}=5 \%$. The outlet of the water flow passage is taken as the outlet of the calculation domain. The number of outlets is three. The water flow direction is perpendicular to the outlet section. Each outlet has an average static pressure of standard atmospheric pressure ( $1 \mathrm{~atm})$ as the outlet condition. The water surfaces of the lead passage and the forebay are free liquid surfaces, and the water level of the forebay does not vary much. The shear stress effect of air on the water surface is neglected. The "rigid cover assumption" is selected in the calculation; that is, the symmetry surface boundary is used. The rest is set as a wall, and a nonslip wall is used for processing.

\section{Analysis of Calculation Results}

3.1. Calculation Evaluation Index. The uniformity of the axial velocity distribution on the inlet section of the flow passage is an important indicator reflecting the velocity distribution on each section. The uniformity of axial velocity distribution is used to characterize the uniformity of the axial (downstream flow direction) velocity distribution of the inlet section of the flow passage. The larger the value is, the closer it is to $100 \%$. This finding shows that the more uniform the axial velocity distribution of the water flow at the inlet section of each flow passage is, the more in line it is with the pump inlet design conditions. The calculation formula is as follows:

$$
V_{\mathrm{au}}=\left[1-\sqrt{\sum_{i=1}^{n} \frac{\left(V_{\mathrm{ai}} / V_{a}-1\right)^{2}}{n}}\right] \times 100 \%,
$$

where $V_{\mathrm{ai}}$ is the axial velocity of each node of the section, $\mathrm{m} /$ $\mathrm{s}$; $V_{a}$ is the average axial velocity of the section, $\mathrm{m} / \mathrm{s} ; n$ is the 


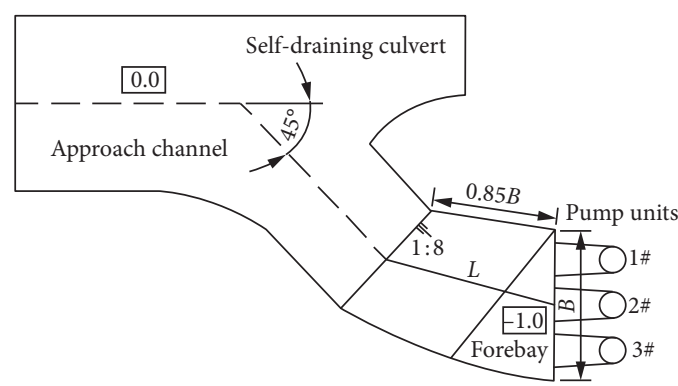

(a)

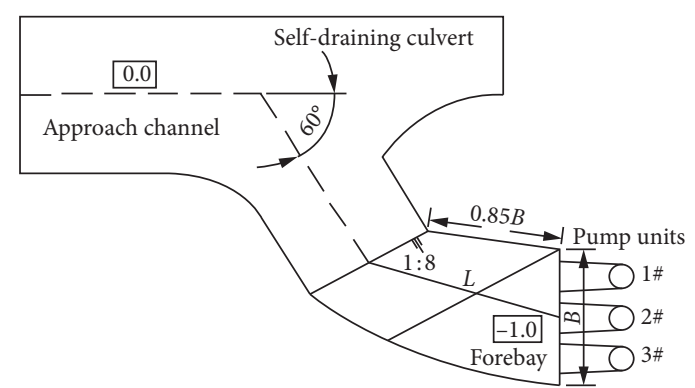

(b)

FIGURE 1: Drawings of pumping station intake buildings under different lateral bending. (a) Layout drawing of the intake building of the pumping station at a $45^{\circ}$ bending. (b) Layout drawing of the intake building of the pumping station at a $60^{\circ}$ bending.

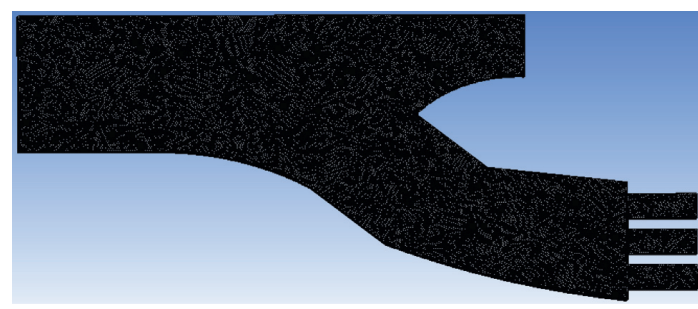

Figure 2: 3D model grid.

number of nodes; and $V_{\text {au }}$ is the uniformity of axial velocity distribution.

The weighted average angle of the inlet section velocity of the passage is an important physical quantity that measures the lateral velocity of the inlet section of each passage. The velocity-weighted average angle is selected to measure the transverse velocity on the inlet section of each flow passage. The closer the value is to $90^{\circ}$, the closer the outlet water flow is perpendicular to the outlet section, and the better the water inlet condition of the pump. The calculation formula is as follows:

$$
\theta=\frac{\sum V_{\mathrm{ai}}\left[90^{\circ}-\arctan \left(V_{\mathrm{ti}} / V_{\mathrm{ai}}\right)\right]}{\sum V_{\mathrm{ai}}},
$$

where $\theta$ is the weighted average angle of velocity at the inlet section of the inlet flow passage; $V_{t i}$ is the lateral velocity of the $i$-th unit grid at the inlet section of the inlet flow passage, $\mathrm{m} / \mathrm{s}$; and $V_{a i}$ is the axial velocity of the $i$-th unit grid at the inlet section of the inlet flow passage, $\mathrm{m} / \mathrm{s}$.

3.2. Feature Section Selection. Seven characteristic sections of the vertical longitudinal section and horizontal section of the forebay are selected for flow field analysis to study the hydraulic characteristics of the forebay of the project, that is, two vertical longitudinal sections perpendicular to the main flow direction and three horizontal longitudinal sections of the forebay surface layer, middle layer, and bottom layer. Figure 4(a) shows the cut vertical longitudinal section, namely, Sections 1-1 and 2.2. The coordinate of Section 1-1 is $X_{1}=0.01 \mathrm{~m}$, which is used as the characteristic section of each passage inlet. The flow field diagram of the 1-1 plane of the section is obtained by numerical simulation to analyze the uniformity of the axial velocity distribution on the inlet section of each flow passage. The coordinates of Section 2.2 are $X_{2}=-0.5 \mathrm{~m}$, and the plane velocity vector diagram of Section 2.2 is obtained by numerical simulation to observe the flow pattern of the main flow in front of the water inlet passage visually.

Figure 4(b) shows the horizontal section taken, namely, surface layer $a-a$, middle layer $b-b$, and bottom layer $c-c$, to study the flow pattern and axial velocity distribution of the forebay surface layer, middle layer, and bottom layer. Surface layer a-a section is $0.05 \mathrm{~m}$ away from the water surface, and the coordinates of the surface layer, middle layer, and bottom layer are $Z_{a}=1.85 \mathrm{~m}, Z_{b}=0.95 \mathrm{~m}$, and $Z_{c}=0.05 \mathrm{~m}$, respectively.

3.3. Numerical Simulation Results of the $45^{\circ}$ Lateral Angle Scheme. Considering that the 3D flow field of numerical simulation is more complicated, the flow velocity vector diagram, streamline diagram, and velocity distribution cloud diagram of characteristic sections are drawn. It is conducive to reflect the flow field of the diversion passage, forebay, and water inlet passage of the lateral intake pump station threedimensionally and intuitively and facilitate the comparative analysis and study of the flow pattern of the entire intake structure.

Figure 5 shows that the water flow in the diversion canal develops smoothly, and the flow pattern is good because the project is a side-inlet forebay. However, after entering the forebay, the main flow of the $1 \#$ inlet passage near the sidewall is severely deviated. While most of the water flows into the water inlet passage, a small part of the water is still in the left side of the forebay (this article uses the flow direction to determine the left and right sides) to form a backflow near the sidewall, touching the sidewall and forming a large-scale backflow area. The flow regime in the return zone is turbulent. Therefore, considering that the water flow in the diversion canal is relatively smooth and the water flow in the forebay is more turbulent, the focus is only on the elaboration and analysis of the flow pattern in the forebay when the lateral angle is $45^{\circ}$.

Figures 6(a)-6(c) show the axial velocity flow field of the surface layer, middle layer, and bottom layer in the scheme with a lateral $45^{\circ}$ bending angle. When water flows from the 
TABLE 1: Calculation results of hydraulic loss under different grid numbers.

\begin{tabular}{|c|c|c|c|c|c|c|}
\hline Number of grids & 1760825 & 2463869 & 2951968 & 3579186 & 4396902 & 5490948 \\
\hline Total inlet pressure $(\mathrm{Pa})$ & 101997 & 101996 & 101995 & 101995 & 101995 & 101994 \\
\hline Total outlet pressure $(\mathrm{Pa})$ & 101732 & 101734 & 101735 & 101736 & 101737 & 101736 \\
\hline Hydraulic loss (m) & 0.02708 & 0.02677 & 0.02662 & 0.02652 & 0.02642 & 0.02633 \\
\hline
\end{tabular}

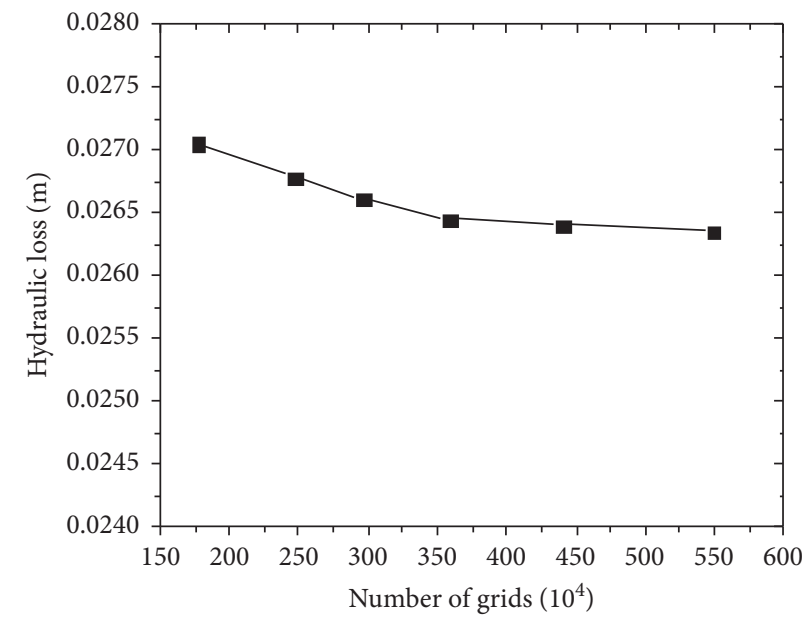

Figure 3: Hydraulic loss under different grid numbers.

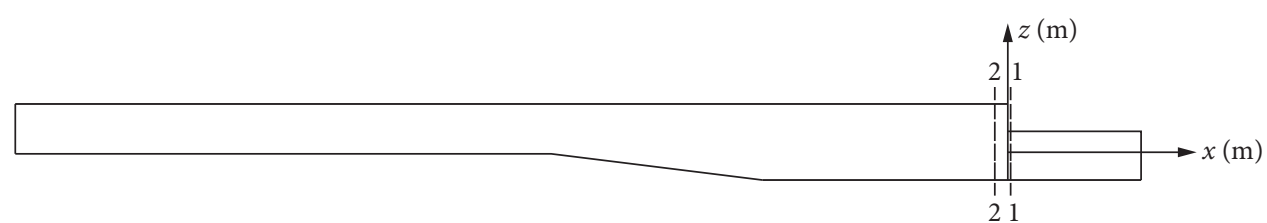

(a)

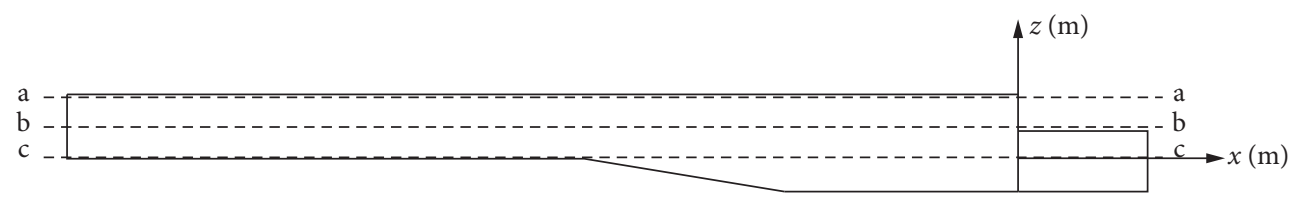

(b)

FIgURE 4: Schematic diagram of the position of each section. (a) Schematic diagram of vertical profile position. (b) Horizontal section position diagram.
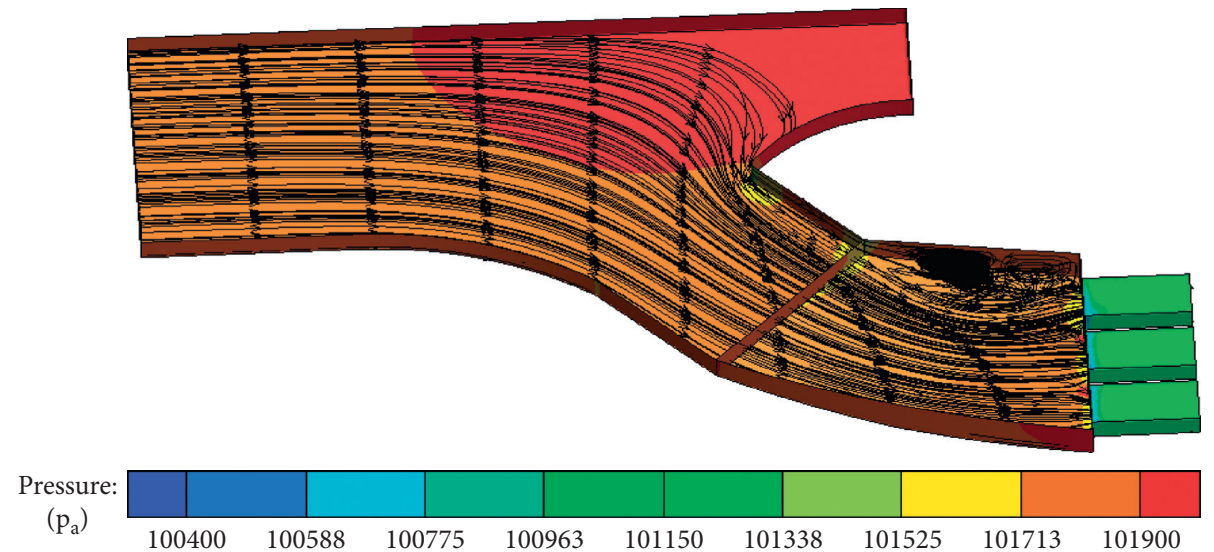

Figure 5: 3D flow field diagram of the lateral $45^{\circ}$ bending angle scheme. 


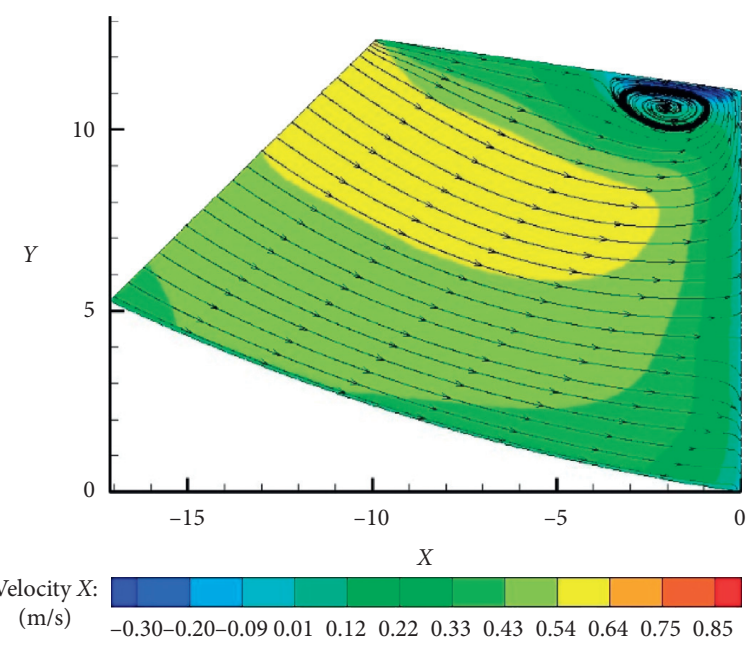

(a)

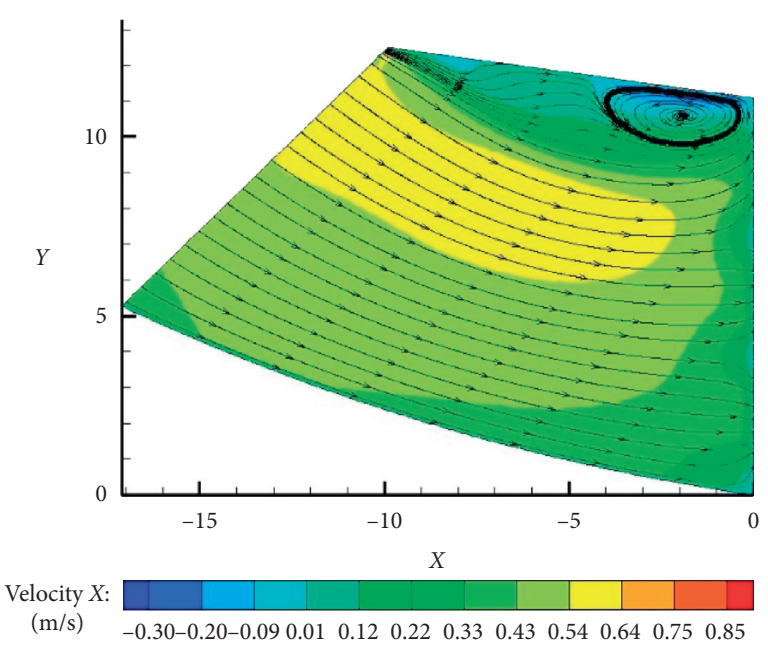

(b)

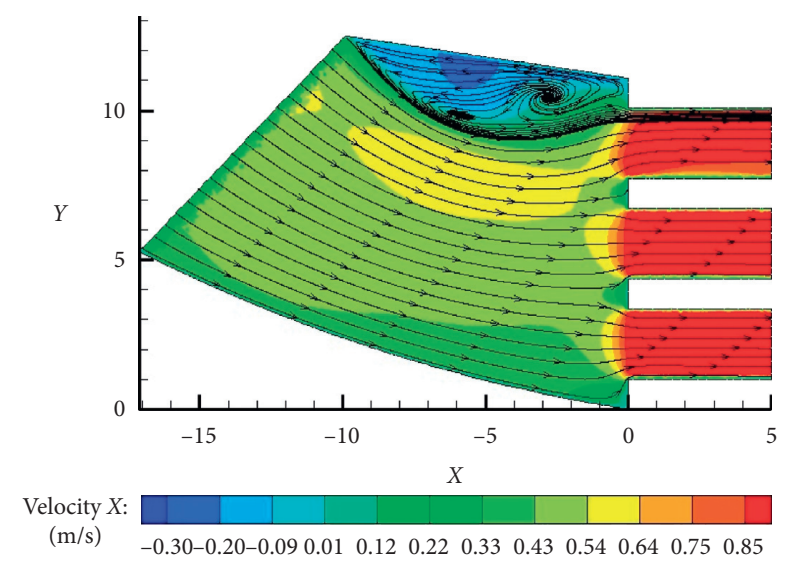

(c)

FIgURE 6: Velocity cloud diagram of the flow field in each horizontal section at a $45^{\circ}$ lateral angle. (a) Surface velocity flow field diagram (b) Midlevel velocity flow field diagram. (c) Bottom velocity flow field diagram.

forebay to the inlet passage, the flow velocity in the middle position of the forebay is relatively large and has a decreasing trend to both sides; most of the fluid can flow smoothly into the inlet passage, but at the left sidewall of the forebay, a backflow zone is evident, and the flow pattern of the reflux zone is extremely high. The reason for this finding is that the transverse velocity of the mainstream is large, and a small part of the fluid contacts the left sidewall, which forms a large range of backflow zone, thus forcing the inflow mainstream to deform and deflect. Comparing and analyzing the flow field maps of each horizontal section show that, during the process from the surface layer to bottom layer, the scope of the reflux area is gradually expanded, and the position of the reflux center is approximately the same. According to the coordinate diagram, the center of the surface layer and middle layer reflux area that is located at $X$ is $-2 \mathrm{~m}, Y$ is $11 \mathrm{~m}$, the bottom layer reflux area center that is located at $X$ is $-3 \mathrm{~m}$, and $Y$ is $11 \mathrm{~m}$. The influent condition of $1 \#$ inlet passage is affected by the backflow zone that occurs before the 1 \# inlet passage.

Figure 7 is a $2-2$ plane velocity vector diagram with a $45^{\circ}$ bending in the lateral direction. The profile position is

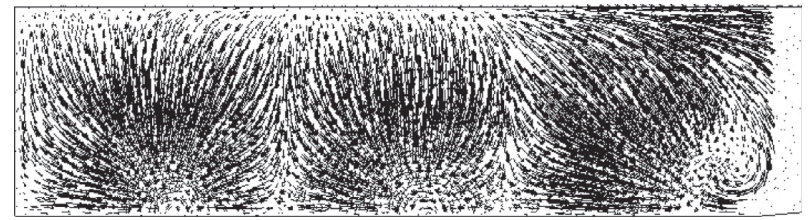

FIGURE 7: 2-2 plane velocity vector diagram with $45^{\circ}$ lateral angle.

$X_{2}=-0.5 \mathrm{~m}$, close to the inlet of the flow passage. The water flow in the forebay has a certain angle of deviation to the left due to the influence of the lateral water inflow. The water flow in the forebay near the inlet of $1 \#$ inlet passage is the most skewed, and the flow is turbulent.

Figure 8 is a $1-1$ plane velocity cloud diagram with a $45^{\circ}$ bending in the lateral direction. The position of the profile is $X_{1}=0.01 \mathrm{~m}$. The inlet flow patterns of $2 \#$ and $3 \#$ inlet passages are better, the velocity distribution is relatively uniform, and the overall distribution is symmetrical. However, the water flow at the inlet of the $1 \#$ passage is severely skewed due to the influence of the backflow in front of the passage. The high-speed area occurs on the left side, 


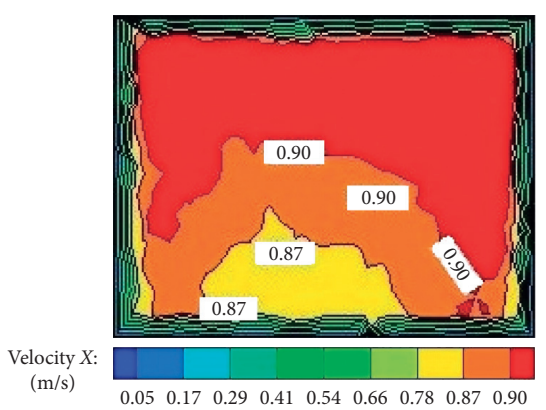

(a)

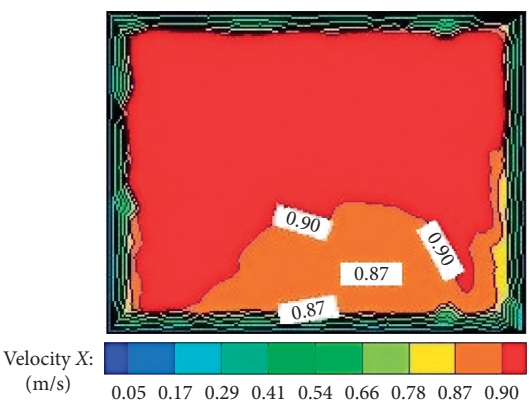

(b)

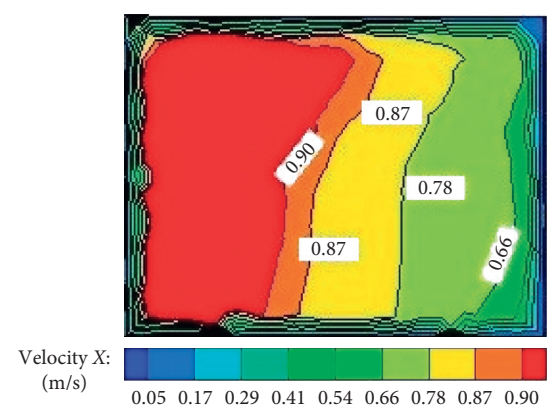

(c)

FIGURE 8: 1-1 plane velocity cloud diagram with $45^{\circ}$ lateral angle (unit: $\mathrm{m} / \mathrm{s}$ ). (a) 3\# passage. (b) 2\# passage. (c) 1\# passage.

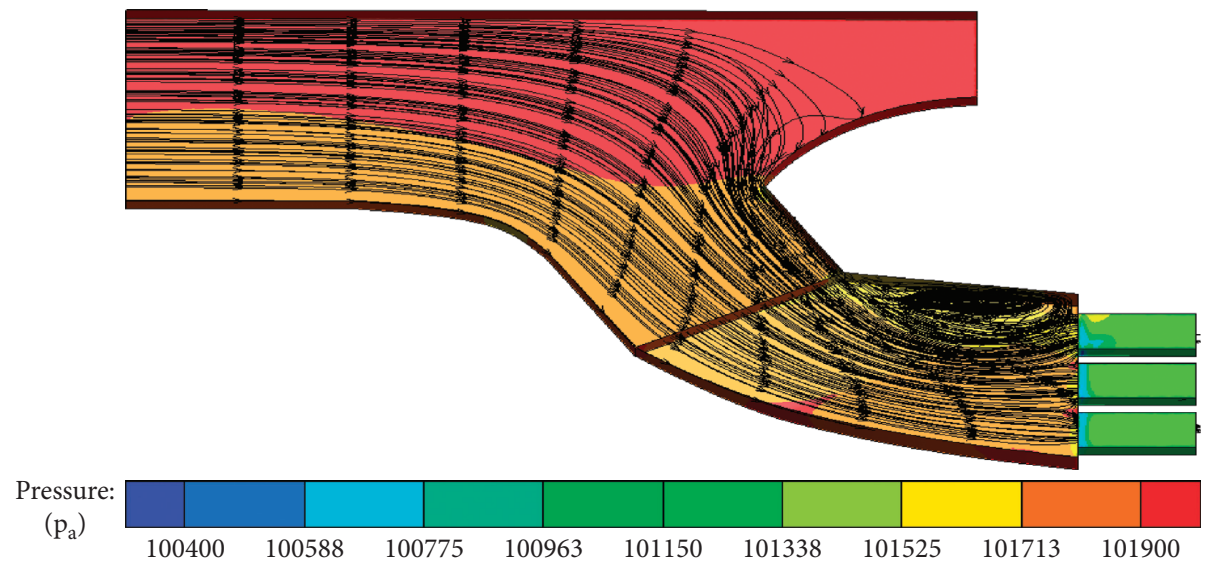

Figure 9: 3D flow field diagram of the lateral $60^{\circ}$ bending angle scheme.

and the flow velocity distribution is extremely uneven. The flow velocity distribution uniformities of the inlet section of $1 \#$, 2\#, and 3\# passages are $83.46 \%, 91.23 \%$, and $91.01 \%$, respectively. The weighted average angles of the inlet section of $1 \#, 2 \#$, and $3 \#$ passages are $69.02^{\circ}, 71.26^{\circ}$, and $70.38^{\circ}$, respectively.

\subsection{Numerical Simulation Results of the Lateral $60^{\circ}$ Bending} Angle Scheme. The position of the characteristic section selected in the plan for a $60^{\circ}$ lateral bending angle is the same as that for a $45^{\circ}$ bending angle. Seven characteristic sections are selected for the analysis of the results. The coordinates of the surface layer, middle layer, and bottom layer are $Z_{a}=1.85 \mathrm{~m}, Z_{b}=0.95 \mathrm{~m}$, and $Z_{c}=0.05 \mathrm{~m}$, respectively; the coordinates of the section perpendicular to the mainstream direction are $X_{1}=0.01 \mathrm{~m}$ and $X_{2}=-0.5 \mathrm{~m}$, respectively. The selected 1-1 section is the three inlet sections of the inlet flow passage to analyze the uniformity and weighted average angle of the axial velocity distribution on the section.

Figure 9 shows that the water flow in the diversion canal is good, but a large-scale backflow zone is observed before the $1 \#$ inlet passage near the left sidewall after entering the forebay. The flow pattern in the recirculation zone is turbulent, and the main flow of the inlet water in front of the $1 \#$ inlet passage is seriously deviated. The comparative analysis shows that the flow pattern of the water flow at a lateral angle of $60^{\circ}$ is the same as that at a lateral angle of $45^{\circ}$, except that the recirculation zone in front of the $1 \#$ inlet passage is larger. Therefore, considering that the water flow in the diversion passage is relatively smooth and the water flow in the forebay is more turbulent, the following only focuses on the elaboration and analysis of the flow pattern in the forebay when the lateral angle is $60^{\circ}$.

Figure 10 shows the axial velocity flow field diagram of each horizontal section when the lateral angle is $60^{\circ}$. Most of the water can flow smoothly into the inlet passage, but a clear backflow zone can be seen on the left sidewall of the forebay. The flow pattern in the return zone is extremely turbulent, and the reason is the same as when the lateral direction is $45^{\circ}$ because the mainstream lateral velocity at this place is relatively large, and a small part of the water flow contacts the left sidewall to form a larger return zone, thereby forcing the main flow of water to deform and deflect. Comparing and analyzing the flow field diagrams of the surface layer, middle layer, and bottom layer show that, from the surface layer to the bottom layer, the scope and position of the recirculation zone are the same, but the center of the recirculation zone moves to the right and forward. According to the coordinate map, the center of the surface and middle recirculation zone is approximately located at $X=-5 \mathrm{~m}$ and $Y=11 \mathrm{~m}$. The bottom recirculation zone center is at $X=-4 \mathrm{~m}$ and $Y=9 \mathrm{~m}$. The flow velocity in the mainstream zone is always greater than that in the recirculation zone. 

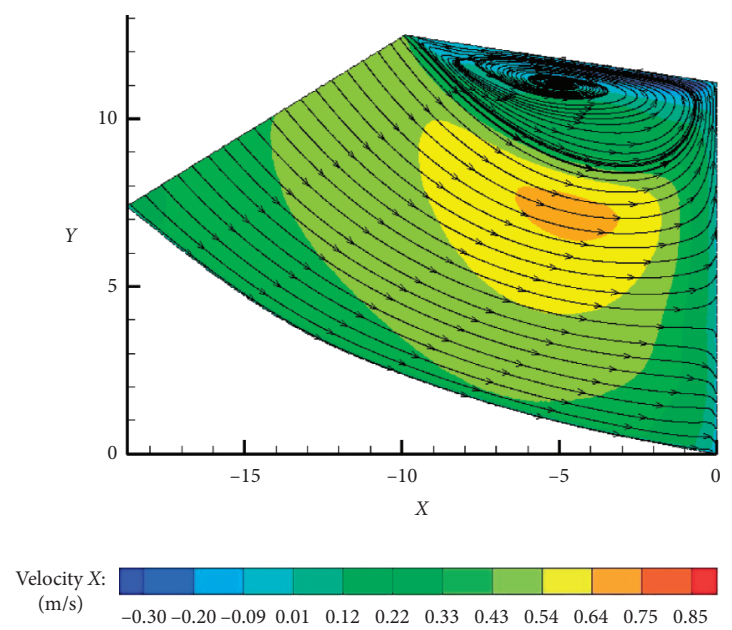

(a)

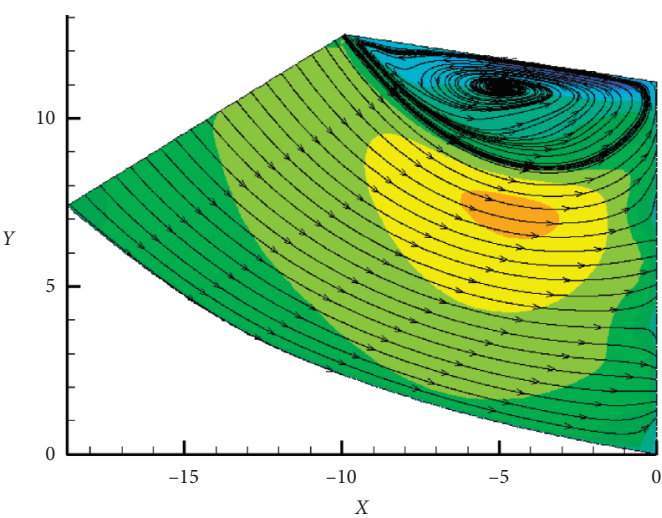

Velocity $X$ : $(\mathrm{m} / \mathrm{s})$

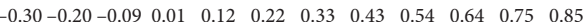

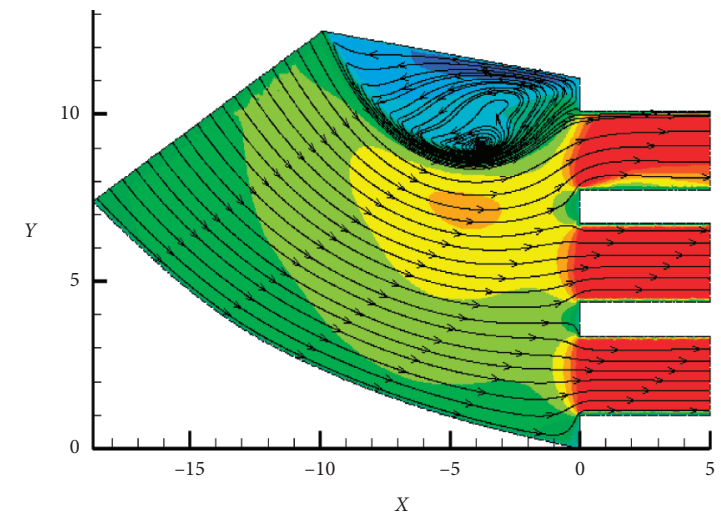

Velocity $X$

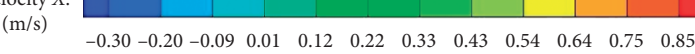

(c)

FIgURE 10: Velocity cloud diagram of the flow field in each horizontal section at a $60^{\circ}$ lateral angle. (a) Surface velocity flow field diagram. (b) Midlevel velocity flow field diagram. (c) Bottom velocity flow field diagram.

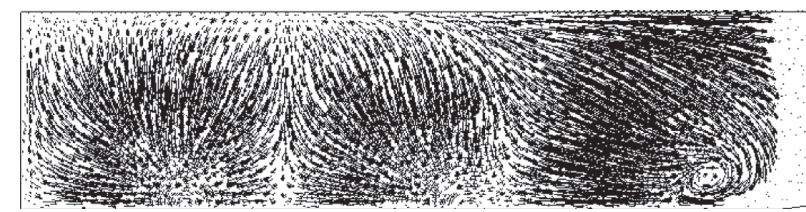

FIGURE 11: Plane velocity vector diagram with a $60^{\circ}$ lateral angle $2-2$.

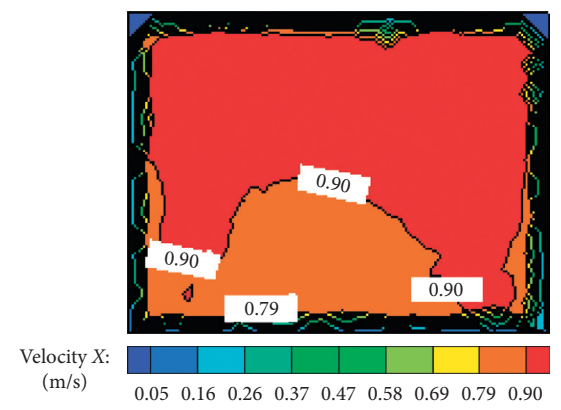

(a)

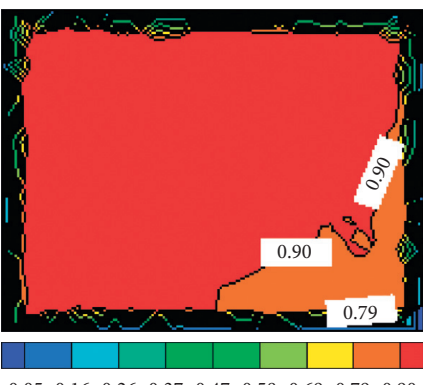

(b)

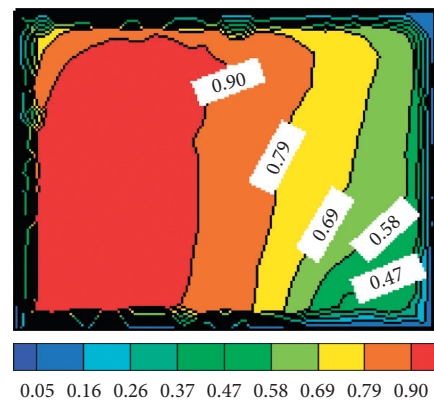

(c)

FIGURE 12:1-1 planar velocity cloud diagram with a $60^{\circ}$ bending angle in the lateral direction (unit: $\mathrm{m} / \mathrm{s}$ ). (a) $3 \#$ passage. (b) $2 \#$ passage. (c) $1 \#$ passage 4 . 
Figure 11 is a $2-2$ plane velocity vector diagram. The profile position is $X_{2}=-0.5 \mathrm{~m}$, near the entrance of the flow passage, and the water in the forebay has an angular deflection to the left due to the influence of the lateral water inflow. The water flow in the forebay near the inlet of $1 \#$ inlet passage is the most skewed, and the flow is turbulent.

Figure 12 is a $1-1$ plane velocity cloud diagram. The section location is $X_{1}=0.01 \mathrm{~m}$. The inlet flow patterns of $2 \#$ and $3 \#$ inlet passages are better, the velocity distribution is relatively uniform, and the overall distribution is symmetrical. However, the water flow at the inlet of the $1 \#$ passage is severely skewed due to the influence of the backflow in front of the passage. The high-speed area occurs on the left side, and the velocity distribution is extremely uneven. The uniformities of the flow velocity distribution of $1 \#, 2 \#$, and $3 \#$ passages are $78.05 \%, 90.78 \%$, and $91.38 \%$, respectively. The weighted average angles of the inlet sections of $1 \#, 2 \#$, and $3 \#$ passages are $63.90^{\circ}$, $71.37^{\circ}$, and $70.41^{\circ}$, respectively.

In summary, the bottom water flow backflow area is the largest for the forebay with $45^{\circ}$ and $60^{\circ}$ lateral angles due to the large lateral velocity of the mainstream at this location, and the large-scale recirculation it forms compresses the streamlines of the mainstream area outward. The recirculation area occurs before the $1 \#$ inlet passage. According to the location where the recirculation zone occurs, the water inlet conditions of the $1 \#$ inlet flow passage are affected, which may endanger the safety of the pump.

\section{Conclusion}

Numerical simulations, including model establishment, setting of boundary conditions, and grid independence analysis, are carried out on the design schemes of the forebay of the inlet pumping station at $45^{\circ}$ and $60^{\circ}$ lateral angles. The turbulence model used is the standard $k-\varepsilon$ model. Through comparative analysis of the numerical simulation results, the following conclusions are drawn:

(1) For the side-inlet pump station, the flow pattern of the water in the front tank is poor during operation, a large-scale return zone is observed, and the flow velocity distribution at the inlet of the $1 \#$ passage is extremely uneven.

(2) Comparing the numerical simulation results of the $45^{\circ}$ lateral bending angle and the lateral $60^{\circ}$ bending angle reveals that the larger the lateral bending angle of the forebay is, the worse the flow pattern of the water flow, and the more unfavorable the pump operation.

(3) Effective engineering measures must be taken to improve the flow pattern in the forebay of the pumping station and ensure the efficient operation of the pumping station.

\section{Data Availability}

The curve data used to support the findings of this study are available from the corresponding author upon request.

\section{Conflicts of Interest}

The authors declare that there are no conflicts of interest regarding the publication of this paper.

\section{References}

[1] X. Zhang, J. Zhou, J. Liang, and H. Zhang, "Experimental research on flow pattern of forebay of side-inlet pumping station," South-to-North Water Transfers and Water Science \& Technology, vol. 14, no. 03, pp. 101-105, 2016.

[2] H. Zhao, F. Yang, C. Liu, S. Chen, and J. He, "Numerical simulation of side-intake flow for fluid meliorating of pumping stations," Water Resources and Hydropower Engineering, vol. 48, no. 07, pp. 79-84, 2017.

[3] J. Zhou, M. Zhao, C. Wang, and Z. Gao, "Optimal design of diversion piers of lateral intake pumping station based on orthogonal test," Shock and Vibration, vol. 2012, no. 9, Article ID 6616456, 2021.

[4] D. Zhang, W. Jiao, L. Cheng et al., "Experimental study on the evolution process of the roof-attached vortex of the closed sump," Renewable Energy, vol. 164, pp. 1029-1038, 2021.

[5] G. Arboleda and M. El-Fadel, "Effects of approach flow conditions on pump sump design," Journal of Hydraulic Engineering, vol. 122, no. 9, pp. 489-494, 1996.

[6] V. P. Rajendran and V. C. Patel, "Measurement of vortices in model pump-intake bay by PIV," Journal of Hydraulic Engineering, vol. 126, no. 5, pp. 322-334, 2000.

[7] C. Hou, "Three-dimensional numerical analysis of flow pattern in pressure forebay of hydropower station," Procedia Engineering, vol. 28, pp. 128-135, 2012.

[8] X. Liu, C. Gao, L. Shi, and W. Liu, "Numerical simulation for fluid meliorating in both forebay and suction bay of pumping station," Journal of Drainage and Irrigation Machinery Engineering, vol. 28, no. 03, pp. 242-246, 2010.

[9] M. Ansar, T. Nakato, and G. Constantinescu, "Numerical simulations of inviscid three-dimensional flows at single- and dual-pump intakes," Journal of Hydraulic Research, vol. 40, no. 4, pp. 461-470, 2002.

[10] L. Cheng, "Computation and analysis of 3-D curved-flow of pumping station forebay and improving of the flow pattern," Journal of Yangzhou University(Natural Science Edition), vol. 2, pp. 64-68, 2002.

[11] C. Liu, L. Cheng, and F. Tang, "Numerical simulation of threedimensional turbulent flow inside a pumping forebay," Transactions of the Chinese Society for Agricultural Machinery, vol. 6, pp. 41-44, 2001.

[12] S. Tang, S. Yuan, and Y. Zhu, "Convolutional neural network in intelligent fault diagnosis toward rotatory machinery," IEEE Access, vol. 8, no. 1, pp. 86510-86519, 2020.

[13] S. Tang, S. Yuan, and Y. Zhu, "Deep learning-based intelligent fault diagnosis methods toward rotating machinery," IEEE Access, vol. 8, no. 1, pp. 9335-9346, 2020.

[14] L. Shi, J. Zhu, F. Tang, and C. Wang, "Multi-disciplinary optimization design of axial-flow pump impellers based on the approximation model," Energies, vol. 13, no. 4, p. 779, 2020.

[15] C. Wang, X. Chen, N. Qiu, Y. Zhu, and W. Shi, "Numerical and experimental study on the pressure fluctuation, vibration, and noise of multistage pump with radial diffuser," Journal of the Brazilian Society of Mechanical Sciences and Engineering, vol. 40, no. 10, p. 481, 2018.

[16] L. Zhou, W. Wang, J. Hang, W. Shi, H. Yan, and Y. Zhu, "Numerical investigation of a high-speed electrical 
submersible pump with different end clearances," Water, vol. 12, no. 4, p. 1116, 2020.

[17] S. Tang, S. Yuan, and Y. Zhu, "Data preprocessing techniques in convolutional neural network based on fault diagnosis towards rotating machinery," IEEE Access, vol. 8, pp. 149487-149496, 2020.

[18] H. Wang, B. Long, Y. Yang, Y. Xiao, and C. Wang, "Modelling the influence of inlet angle change on the performance of submersible well pumps," International Journal of Simulation Modelling, vol. 19, no. 1, pp. 100-111, 2020.

[19] H. Wang, Z. Qian, D. Zhang, T. Wang, and C. Wang, "Numerical study of the normal impinging water jet at different impinging height, based on wray-agarwal turbulence model," Energies, vol. 13, no. 7, p. 1744, 2020.

[20] C. Wang, W. Shi, X. Wang et al., "Optimal design of multistage centrifugal pump based on the combined energy loss model and computational fluid dynamics," Applied Energy, vol. 187, pp. 10-26, 2017.

[21] L. Zhou, K. Deshpande, X. Zhang, and R. K. Agarwal, "Process simulation of Chemical Looping Combustion using ASPEN Plus for a mixture of biomass and coal with various oxygen carriers," Energy, vol. 195, Article ID 116955, 2020.

[22] L. Zhou, C. Han, L. Bai, W. Shi, and Agarwal, "Numerical and experimental study of multiphase transient core-annular flow patterns in a spouted bed," ASME Journal of Energy Resource Technology, vol. 142, no. 9, Article ID 092104, 2020.

[23] H. Wang, B. Long, C. Wang, C. Han, and L. Li, "Effects of the impeller blade with a slot structure on the centrifugal pump performance," Energies, vol. 13, no. 7, p. 1628, 2020.

[24] H. Wang, Q. Hu, Y. Yang, and C. Wang, "Performance differences of electrical submersible pump under variable speed schemes," International Journal of Simulation Modelling, vol. 20, no. 1, pp. 76-86, 2021. 